\title{
Acetyl-11-keto- $\beta$-boswellic acid Restrains Inflammation and Extracellular Matrix Degradation of Osteoarthritis via Suppression of NF-KB Pathway
}

\section{Jing Zhou}

The Affiliated Suzhou Hospital of Nanjing Medical University

\section{Zeyu Han}

Capital Medical University

\section{Xueyan Li}

Nanjing Medical University

\section{Zhiqiang Shao}

The Affiliated Suzhou Hospital of Nanjing Medical University

\section{Yinhua Qian}

The Affiliated Suzhou Hospital of Nanjing Medical University

\section{Lang Bai}

The Affiliated Suzhou Hospital of Nanjing Medical University

Qibin Han

The Affiliated Suzhou Hospital of Nanjing Medical University

\section{Xiaoyu Zhang}

The Affiliated Suzhou Hospital of Nanjing Medical University

\section{Qi Chen}

The Affiliated Suzhou Hospital of Nanjing Medical University

\section{Maofeng Gao}

The First Affiliated Hospital of Soochow University

\section{Yi Xue}

Changshu Hospital, Nanjing University of Traditional Chinese Medicine

\section{Dechun Geng}

The First Affiliated Hospital of Soochow University

\section{Xing Yang ( $\nabla$ xingyangsz@126.com )}

The Affiliated Suzhou Hospital of Nanjing Medical University

\section{Yuefeng Hao}

The Affiliated Suzhou Hospital of Nanjing Medical University 
Keywords: acetyl-11-keto- $\beta$-boswellic acid, osteoarthritis, inflammation, extracellular matrix, NF-kB

Posted Date: November 23rd, 2022

DOI: https://doi.org/10.21203/rs.3.rs-1234256/v2

License: (c) (1) This work is licensed under a Creative Commons Attribution 4.0 International License. Read Full License 


\section{Abstract}

Background. Mechanical stress along with inflammation play causative roles in the development of osteoarthritis (OA), which decreases the quality of life and causes economic loss. Inflammation and extracellular matrix (ECM) degradation have been identified as key factors in the development of OA. As the main active component in frankincense, acetyl-11-keto- $\beta$-boswellic acid (AKBA) has been shown to have positive effects on inflammation. However, the effects of AKBA in cartilage inflammation and ECM degradation are currently elusive.

Methods. We demonstrated the role of inflammation and ECM degradation in the pathogenesis of OA and determined the protective effect of AKBA on both Hulth-Telhagrat OA model and lipopolysaccharide (LPS)-induced rat chondrocytes.

Results. We found increased inflammatory expression and decreased ECM expression in OA model cartilage and LPS-induced chondrocytes. Meanwhile, the protective effect of AKBA and its inhibitory effects on inflammation as well as ECM-related markers were also observed in the rat Hulth-Telhag model. Furthermore, activation of NF-KB attenuated nuclear p65 protein levels in chondrocytes upon LPS stimulation. In addition, AKBA was found to subsequently reversed the LPS-induced activation of NF- KB signal and inflammation-related ECM degradation in chondrocytes.

Conclusions. Suppression of NF-KB pathway activation by AKBA restrains OA development via inhibition of inflammation and ECM degradation. AKBA is a promising therapeutic agent for the treatment of OA.

\section{Background}

Osteoarthritis (OA), a quite common chronic and degenerative disease in orthopedics, decreases the quality of life and causes economic loss globally.(Glyn-Jones et al., 2015) The incidence of OA is continuously increasing because of aging, genetic factors, inflammation, obesity, and biological and biomechanical factors.(Reyes et al., 2016) (Loeser et al., 2016)The development of OA cannot be reversed and terminated under current technical conditions. The specific principle is non-drug therapy combined with medication palliative treatment is mainly applied to relieve symptoms, if necessary, surgical treatment.(Quicke et al., 2022) However, high cost, prosthesis loosening, secondary revision of wear, unnatural angle of movement, and limited movement limit the surgical treatment. Currently, there are various drugs used to treat OA, of which nonsteroidal anti-inflammatory drugs (NSAIDs) are the most widely used. However, long-term use of NSAIDs can confer negative effects on coagulation, digestion, and urinary systems.(Zeng et al., 2018) Therefore, exploring the pathogenesis and treatment of OA could be of great practical significance.

Biological and biomechanical factors such as mechanical stress and inflammation disrupt the balance between anabolism and catabolism of chondrocytes, extracellular matrix (ECM), and subchondral bone, leading to degenerative fibrosis of joint cartilage and subchondral bone sclerosis.(Quicke et al., 2022) (Xia et al., 2014) Inflammation and ECM degradation represent hallmarks of OA progression, resulting in 
the pathological changes of OA. Elevated inflammatory factors, such as interleukin (IL)-1 $\beta$, IL-6, and tumor necrosis factor (TNF)-a, can promote the expression of matrix metalloproteinases (MMPs) and other catabolic enzymes to destruct the ECM, which have been reported to upset the balance between anabolism and catabolism in normal cartilage.(Lieberthal et al., 2015) (Lieberthal et al., 2015) (Carballo et al., 2017) As essential components of the ECM, the expression level of aggrecan and collagen II (col II) is an important indicator of the degree of cartilage degeneration and its decreased activity can lead to ECM regeneration disorder, eventually causing cartilage degeneration.(Jiang, 2022)

Frankincense, the resin exuded from the bark of Boswellia carterii birdw, which belongs to Oleaceae plants, has long been recognized as a traditional medicine in India and China.(Ning et al., 2018) Studies have found that acetyl-11-keto- $\beta$-boswellic acid (AKBA) can significantly alleviate the symptoms of OA. (Sengupta et al., 2008) As the most active ingredient in frankincense, AKBA has become the primary research object with stronger biological activity than 11-keto-ß-boswellic acid (KBA).(Roy et al., 2019) (Miscioscia et al., 2019) Multiple studies have found that AKBA has anti-inflammatory, analgesic, antiulcer, anti-oxidation, anti-tumor, anti-osteogenic inhibition, immunomodulatory, and lipid regulatory capabilities.(Meka et al., 2017) (Tambe et al., 2019) (Rajabian et al., 2020) (Li et al., 2018) (Xiong et al., 2019) Whereas few studies have associated AKBA with cartilage metabolism, others have indicated that AKBA could relieve the symptoms of OA. Moreover, the specific mechanism of AKBA on the development of OA has not been investigated, which restricts the further application of AKBA and its derivatives.

Herein, we probed the association between AKBA intervention and surgery and lipopolysaccharide (LPS)induced inflammation and ECM degradation. Hulth-Telhag OA model was used in vivo and LPS was used to induce inflammation in vitro. Our results confirmed that AKBA intervention attenuated inflammation and ECM degradation. The inhibition of inflammation and ECM degradation was achieved through AKBAmediated inhibition of the NF-KB signaling pathway. In a nutshell, these results demonstrate that AKBA is a promising therapeutic approach for the prevention and treatment of OA.

\section{Materials And Methods \\ 2.1 Ethics statement}

Animal welfare and experimental procedures were carried out in accordance with $t$ international guidelines on animal welfare ethics, and were approved by the Ethics Committee of the First Affiliated Hospital of Soochow University.

\subsection{Compounds and reagents}

AKBA (CAS No.: 67416-61-9, purity: 99.71\%) was purchased from MCE (Shanghai, China). N-3-oxododecanoyl-L-Homoserine lactone (3-O-C12-HSL, Cas No.:168982-69-2, purity: 98.00\%) was purchased from Apexbio (Houston, USA).

\subsection{Animals and experimental design in vivo}


Approximately 24 eight-week-old Sprague Dawley (SD) male rats were raised in a standard environment and were randomly kept in cages, where water and fodder supply were sufficient. The Hulth-Telhag OA model was established. After one week of adaption feeding, SD rats were randomly divided into four groups: control, vehicle, low-AKBA, and high-AKBA groups ( $n=6 /$ group). Except for the control group, the rats in the other three groups received pentobarbital ( $40 \mathrm{mg} / \mathrm{kg}$, intraperitoneally) for anesthesia. After anesthesia, both lower extremity operation area of the rat was shaved, followed by repeated penicillin $G$ $(80000 \mathrm{U} / \mathrm{rat})$ intramuscular administration for 3 days. The operation area was disinfected per the aseptic operation regulations, the anterior medial longitudinal skin incision was taken, the knee joint was exposed, and the medial side was cut off. The medial collateral ligament, the anterior and posterior cruciate ligaments, and the medial meniscus were removed. The articular cartilage surface was protected during the operation. The joint cavity and epidermis were sutured layer by layer using a $3-0$ silk thread. The affected limb was not fixed after the operation. The experimental animals were returned to the animal room to continue feeding. One week after modelling, the rats were administered with $0.5 \mathrm{ml} / \mathrm{kg}$ saline, as well as 2 and $8 \mathrm{mg} / \mathrm{kg}$ AKBA solution by gavage every other day. All experimental animals were sacrificed after 6 weeks and samples were taken for analysis.

\subsection{Micro-computed tomography (micro-CT) and reconstruction}

The rats were sacrificed after 6 weeks by intraperitoneal injection of excess pentobarbital sodium, and the knee joint ( $n=6$ /group) were collected and fixed with $10 \%$ paraformaldehyde. A SkyScan 1176 CT machine (BRUKER, Belgium) was used for CT. The scanning parameters were $60 \mathrm{kV}$ source voltage and $170 \mu \mathrm{A}$ source current, and the resolution was set at $9 \mu \mathrm{m}$. The 3D reconstruction used the procedure provided by the factory. Bone trabecular bone density $\left(\mathrm{Tb} . \mathrm{BMD}, \mathrm{mg} / \mathrm{cm}^{3}\right)$, bone volume fraction (BV/TV, $\%$ ), number of trabecular bone ( $\mathrm{Tb} . \mathrm{N}, \mathrm{mm}^{-1}$ ), the thickness of trabecular bone ( $\mathrm{Tb}$. Th, $\mu \mathrm{m}$ ), trabecular bone separation (Tb. Sp, mm), and connection density (Conn. D, $\mathrm{mm}^{-1}$ ) were analyzed with SkyScan software.

\subsection{Histologic analysis}

For decalcification, samples from each group were placed into $10 \%$ ethylenediaminetetraacetic acid (EDTA) for 4 weeks, paraffin-embedded samples were cut into 6 - $\mu \mathrm{m}$ slices and staining procedures, including hematoxylin and eosin (H\&E), safranin O-fast green, and tartrate-resistant acid phosphatase (TRAP) staining, were performed according to the manufacturer's protocols. An AxioCam HRc microscope (Carl Zeiss, Jena, Germany) was used to capture the images. Morphological scores of H\&E and safranin 0 -fast green staining were given to sections under a micro-imaging system according to the International Academy of Osteoarthritis Research (OARSI) scores, and three readers were arranged for each section to obtain the average as the final score. After TRAP staining was completed, 5 fields of vision under the cartilage or above the tide line were randomly selected, the number of TRAP-positive cells, and the proportion of osteoclast surface (OcS/BS, \%) were counted using the BIOQUANT OSTEO (BIOQUANT, USA). 


\subsection{Immunohistochemical (IHC) analysis}

Expression levels of IL-1 $\beta, \mathrm{IL}-6, \mathrm{TNF}-\mathrm{a}, \mathrm{MMP} 13, \mathrm{col}$, col , IKKa/ $\beta$, IKBa, and p65 in the knee joint was examined using IHC analysis with corresponding primary antibodies (Abcam, ab9722, ab9324, ab9739, ab39012, ab34712, ab39012, ab178870, ab32518, and ab16502). Briefly, the sections were dewaxed for $1 \mathrm{~h}$ with xylene and then subjected to gradient hydration, antigen retrieval with hyaluronidase $\left(1 \mathrm{~h}, 37^{\circ} \mathrm{C}\right)$, and pepsin ( $25 \mathrm{~min}, \mathrm{RT})$. Next, the sample sections were blocked with bovine serum albumin (BSA, $1 \mathrm{~h}$, room temperature (RT)), followed by incubation with correspondent primary antibodies $\left(12 \mathrm{~h}, 4^{\circ} \mathrm{C}\right)$. After washing with PBS, sections were incubated with secondary antibodies (30 min, RT). The chromogenic reaction was induced by a DAB horseradish peroxidase color development kit (Beyotime, Shanghai, China). In the semi-quantitative analysis, positive cell counts were randomly selected under the microscope to count positive cells and non-positive cells, and the proportion of positive cells was obtained. The average number was used as the proportion of positive cells in the section. The average optical density analysis was completed with Image Pro-Plus 6.0 software, and uniform white balance and exposure time settings used in the screenshot were adopted. Because col II is only concentrated on the surface of the cartilage, the only semi-quantitative analysis was performed on the surface of the cartilage.

\subsection{Cell culture}

Rat primary articular chondrocytes (Cat NO.: CP-R092) was purchased from Procell (Wuhan, China). After digestion by $0.25 \%$ trypsin, chondrocytes were cultured in F-12 medium mixed with $10 \%$ fetal bovine serum (FBS) and $1 \%$ penicillin/streptomycin in a regular incubator $\left(37^{\circ} \mathrm{C}, 5 \% \mathrm{CO}_{2}\right)$. The medium was changed every 3 days. Cells subculturing was performed when the cells reached $80-90 \%$ confluency after washing and digestion. Cells were then treated with AKBA in low $(2 \mu \mathrm{M})$ or high $(8 \mu \mathrm{M})$ concentrations.

\subsection{Cell viability assay}

Chondrocytes were seeded ( $5 \times 10^{3} /$ well in $0.5 \mathrm{ml}$ of F-12 medium) in 96-well plates and cultured with a range of concentrations of $\operatorname{AKBA}(0,2,4,8,16,32,64$, and $128 \mu \mathrm{M})$ for 24,48 , and $72 \mathrm{~h}$ after adherence. After reaching the corresponding time point, the PBS-washed cells were incubated with a cell counting kit8 (CCK-8) (Dojindo, Shanghai, China) $\left(3 \mathrm{~h}, 37^{\circ} \mathrm{C}\right)$ and the optical density (OD) of chondrocytes at $450 \mathrm{~nm}$ was determined. The cell viability to control was calculated using the equation: Cell viability to control (\%) = OD drug-treated group $/ O D$ control group.

\subsection{Western blot analysis}

After treatment, cell proteins were extracted using radioimmunoprecipitation assay (RIPA) buffer with protease inhibitors and phosphatase inhibitors. The protein concentration was determined by a nucleic acid-protein quantometer (Thermo Scientific, Shanghai, China), and then separated by sodium dodecyl sulfate-polyacrylamide gel electrophoresis (SDS-PAGE). The resolved proteins were transferred to a polyvinylidene difluoride (PVDF) membrane and blocked with blocking solution (1 h, RT). Next, PVDF membranes were incubated with corresponding primary antibodies (overnight, $4^{\circ} \mathrm{C}$ ), followed by 
incubation with the secondary antibody $\left(1 \mathrm{~h}, 4^{\circ} \mathrm{C}\right)$. The reactive protein bands were detected by the Western blot detection kit. Image analysis was performed using Image $\mathrm{J}$ software.

\subsection{Quantitative real-time (qRT)-PCR}

After treatment, total RNA was extracted using Beyozol reagent (Beyotime, Shanghai, China). The quality of RNA was confirmed by measuring the absorbance of the A260/A280 and A260/A230 ratio in the range of 1.8-2.0, respectively. cDNA was synthesized with MonScript ${ }^{\text {TM }}$ RTIII Super Mix with dsDNase (Monad, Wuhan, China) following the manufacturer's protocols. Subsequently, MonAmp ${ }^{\text {TM }}$ ChemoHS qPCR Mix (Monad, Wuhan, China) was used to perform qRT-PCR. The parameters were as follows: $95.0^{\circ} \mathrm{C}$ for 10 min, 40 cycles of $95.0^{\circ} \mathrm{C}$ for $10 \mathrm{~s}, 60.0^{\circ} \mathrm{C}$ for $10 \mathrm{~s}$, and $72.0^{\circ} \mathrm{C}$ for $10 \mathrm{~s}$. All samples were analyzed in triplicate and three independent experiments. The comparative $2^{-\Delta \Delta C q}$ method was used for analysis. GraphPad Prism 8 software was used to perform statistical analysis and draw graphs. The primer sequences are shown in Table 1.

Table.1

The primer sequences

\begin{tabular}{lll}
\hline \multicolumn{3}{l}{ Primer sequence } \\
\hline mRNA & Forward(5'-3') & Reverse(3'-5') \\
IL-1 $\beta$ & GCCCATCCTCTGTGACTCAT & AGGCCACAGGTATTTTGTCG \\
IL-6 & TTGGGAAGGTTACATCAGATC & GGGTTGGTCCATGTCAATTT \\
TNF- $\alpha$ & GATGGACTCACCAGGTGAG & CTCATGGTGTCCTTTCCAGG \\
Aggrecan & GAGAAGGAGGTAGTGCTGCTGG & GATGCACAAGGTAATGTCTCGGTA \\
collagen II & TCCTAAGGGTGCCAATGGTGA & GGACCAACTTGCCTTGAGGAC \\
MMP-1 & TAGGTGTGGGGTGCCTGATG & GCTCTCTCGATGGCGTTTCT \\
MMP-3 & CCTCTATGGACCTCCCACAGAATC & GGTGCTGACTGCATCGAAGGACAAA \\
MMP-13 & CTGGCCTGCTGGCTCATGCTT & CCTCAGAAAGAGCAGCATCGATATG \\
GAPDH & ACTGGCGTCTTCACCACCAT & AAGGCCATGCCAGTGAGCTT \\
\hline
\end{tabular}

\subsection{Immunofluorescence (IF) assay}

Chondrocytes climbing slices were incubated with or without LPS $(1 \mu \mathrm{g} / \mathrm{ml})$ for $24 \mathrm{~h}$, and then coincubated with or without AKBA $(8 \mu \mathrm{M})$ for $24 \mathrm{~h}$. PBS-washed slices were fixed in $4 \%$ paraformaldehyde solution (15 min, RT), washed thrice with PBS, and then treated with $0.1 \%$ TritonX-100 (5 min, RT). Climbing slices were then shifted into a wet box, blocked with blocking solution $\left(1 \mathrm{~h}, 37^{\circ} \mathrm{C}\right)$, and then incubated with primary antibodies (MMP13 (1:200) and p-p65 (1:200)) diluted with their dilutions for 12 $h$ at $4^{\circ} \mathrm{C}$. After incubating with iFluor ${ }^{\mathrm{TM}} 488$ goat anti-rabbit IgG antibody (1:1000, AAT Bioquest, Calif, 
USA) in darkness for $1 \mathrm{~h}$, DAPI (4',6-diamidino-2-phenylindole) was used to stain the cell nuclei. The slices were dried and sealed by an anti-fluorescence quenching agent and imaged using confocal microscopy (Carl Zeiss AG, Jena, Germany) after aspirating the liquid from the climbing slices.

\subsection{Statistical analysis}

The quantitative data were expressed as the means \pm standard deviation (SD). GraphPad Prism 8 (GraphPad Software Inc, USA) was utilized for data analysis and statistical mapping. The two samples were compared using an independent sample t-test. A P-value $<0.05$ was considered statistically significant.

\section{Results}

\subsection{AKBA treatment had positive effects on the development of a surgery-induced OA rat}

To explore the potential effects of AKBA in the development of OA, a Hulth-Telhag model was established (Fig. S1). AKBA was administered by gavage every other day for 6 weeks after the operation. Micro-CT, H\&E staining, safranin O-fast green staining, TRAP staining, and OARSI scores were used to observe the cartilage structure, the morphological appearance of the cartilage cells, and osteoclast activity difference in the control, vehicle, and low/high-AKBA groups. Micro-CT analysis indicated that specimens in the controls had a normal joint space and bone mineral density. The vehicle group had narrowing of the joint space and hyper osteogeny; however, in the AKBA-treatment group, these were reversed (Fig. 1(A)). Bone morphometric analysis was performed to further quantify and compare the parameters of the trabecula. The results showed that several parameters of the bone trabecula, such as BV/TV, Tb. N, Tb. Th, Conn. D, etc., decreased in the solvent group compared to the controls while Tb. Sp increased and the difference was significant (Fig. 1(B)-(G)). After AKBA treatment, the decrease of BV/TV, Tb. N, and Conn. D decreased and the increase of Tb. Sp decreased in the low-AKBA group. Collectively, these statistics showed that AKBA eased surgery-induced changes in the subchondral bone.

After treatment, specimens were counterstained with H\&E and safranin O-fast green staining (Fig. 1(H)). Sections were examined under a microscope based on the OARSI scoring system (Fig. 1(I)-(K)). The control group grade averaged 0.1667 , with a staged interval of $0-1.0$, the cartilage surface was intact, and the cell structure was normal. The solvent group grade averaged 3.750, with a staged interval of 2.54.5, with marked cartilage matrix loss. Large cysts were formed in the area, chondrocytes clustered, some cells were significantly enlarged, cartilage structure was disordered, and degeneration was substantial. In the low-AKBA group, the average grade was 2.750 and the stage interval was 2.0-3.5. There was a phenomenon of focal deep staining. Occasionally, cracks penetrated deep into the cartilage area, and there was chondrocytes clustering and hypertrophy. The high-AKBA group had an average grade of 1.5 and a stage interval of 1.0-2.0. The surface of the cartilage was discontinuous and there was focal staining. It was enhanced, but cell hypertrophy and clustering phenomenon was not evident, and the 
degree of degeneration was lighter. The solvent composition level was significantly different from the controls $(P<0.01)$. The improvement of the solvent composition level was significant $(P<0.05)$ and the difference between the low-AKBA and the high-AKBA groups was also significant $(P<0.01)$. It showed that AKBA could alleviate the depth of arthritis invading cartilage. In terms of staging, there was a significant difference between the vehicle group and the control group $(P<0.01)$ but there was no significant difference in staging between the low-AKBA group and the high-AKBA. Because the stage mainly shows the size of the affected area of the joint, the effect of AKBA on the size of the affected area was relatively small. In terms of the overall score, the vehicle group was also significantly different from the controls $(P<$

Next, we performed TRAP staining on the specimens to identify differences in osteoclast activity between different groups. The results showed that the osteoclast was more active in the vehicle group than in the controls (Fig. S2(A)), which is consistent with the findings of micro-CT. About the quantitative analysis, the vehicle group increased significantly compared to the control group (Fig. S2(B) and (C)) both in the TRAP-positive cell count and OcS/BS, suggesting that the osteoclast was very active in the early stage of OA. In the AKBA intervention group, the osteoclast count in the low-AKBA group decreased compared with OcS/BS in the vehicle group, which were $44.9 \%(P<0.05)$ and $32.0 \%(P<0.05)$, respectively, and the difference was significant. Interestingly, this effect was also evident in the high-AKBA group, reaching $67.6 \%(P<0.01)$ and $77.9 \%(P<0.01)$. However, between the low-AKBA and the high-AKBA groups, the TRAP-positive cell count did not show a significant difference, but there were significant differences in OcS/BS, showing a dose-dependent effect (Fig. S2(B) and (C)).

\subsection{AKBA treatment repressed inflammation and ECM degradation in surgery-induced $O A$ rat}

To delve deeper into whether AKBA treatment could attenuate inflammation and ECM degradation in surgery-induced $O A$ rats, specimens were immunoassayed to evaluate the expression of inflammatory and ECM biomarkers. AKBA intervention significantly eased the expression levels of inflammatory markers in surgery-induced OA rats (Fig. 2(A)). However, more IHC-positive cells were observed in the vehicle group compared with the other groups. These results implied that the removal of the medial collateral ligament, the anterior and posterior cruciate ligaments, and the medial meniscus enhanced the expression of inflammatory markers, while AKBA intervention reversed the results (Fig. 2(B)-(D)).

We selected common cartilage degeneration markers MMP13 and major cartilage matrix components col and (Fig. 2(E)) for IHC, of which MMP13 was closely related to cartilage degeneration and its expression level was strongly associated with the level of cartilage degeneration. The IHC analysis showed that the average proportion of MMP13-positive cells in the vehicle group increased by nearly 1.5 times compared with the controls, and the degeneration performance was significant. The proportion of positive cells in the vehicle group was higher than that in the high-AKBA group at the same time. The average values decreased by $35.9 \%$ and $53.4 \%$, respectively, and the difference was significant $(P<0.01)$. Similarly, the difference between the high-AKBA group and the low-AKBA group was also significant. We 
noticed that in the control group, col II was mainly concentrated on the surface of cartilage, where there was almost no col I. Col I content, however, was higher below the surface layer. In the vehicle group, we found that col II staining depth of the cartilage surface layer decreased significantly and more col I appeared, suggesting severe matrix loss and active fibrogenesis. In the AKBA treatment groups, the staining depth of col I increased, while the staining depth of col I on the surface of cartilage decreased (Fig. 2(E)). Semi-quantitative analysis (Fig. 2(G)-(J)) showed that col II-positive cells and expression decreased in the state of arthritis, while the expression of col I and the number of positive cells increased. However, col expression increased whereas col expression AKBA treatment alleviated LPS-induced inflammation and ECM degradation in chondrocytes

The chemical structure of AKBA and 3-O-C12-HSL were showed in Fig. S3(A) and (D). To determine the cytotoxicity of AKBA and 3-0-C12-HSL on rat chondrocytes, chondrocytes were cultured in a series of concentrations $(0,1,2,4,8,16,32,64$, and $128 \mu \mathrm{M})$ of AKBA for 1,2 , and 3 days separately, before cell viability was detected by CCK-8 assay. The results indicated that AKBA and 3-O-C12-HSL affected the viability of chondrocytes when the concentration was $>8 \mu \mathrm{M}$ (Fig. S3(B) and (C)) and $>16 \mu \mathrm{M}$ (Fig. S3(E) and $(F))$ on the third day. This shows that AKBA at 2 and $8 \mu \mathrm{M}$ can be utilized in the subsequent experiments.

The mixed chondrocytes were pretreated by $1 \mu \mathrm{g} / \mathrm{ml}$ LPS for $24 \mathrm{~h}$ followed by treatment with low $(2 \mu \mathrm{M})$ or high $(8 \mu \mathrm{M})$ concentrations of AKBA for $24 \mathrm{~h}$ to evaluate the potential inflammatory effects of AKBA in LPS-induced chondrocytes. Western blotting and qRT-PCR were used to test the protein and mRNA levels of inflammatory biomarkers. Although LPS-stimulated production of IL-1 $\beta$, IL-6, and TNF-a was significantly upregulated compared with the controls, these biomarkers were reversed by AKBA at $2 \mu \mathrm{M}$ and $8 \mu \mathrm{M}$ in a dose-dependent trend (Fig. 3(A)-(D)). Meanwhile, there was significant downregulation of inflammatory mRNA levels (Fig. 3(E)-(G)). The results indicated that AKBA could suppress the LPSinduced inflammatory response in chondrocytes in a concentration-dependent manner.

IF analysis was performed to visualize infected cells, which significantly reversed the downregulation of col II (Fig. 3H). We investigated the effects of AKBA on aggrecan, col II, and MMPs mRNA and protein expression to confirm whether AKBA could protect against LPS-induced matrix degradation. The results showed that AKBA enhanced aggrecan and col II protein (Fig. 3(I)-(K)) and mRNA (Fig. 3(O) and (P)) expression in LPS-induced chondrocytes, which was dependent on AKBA concentration. As expected, the expression levels of MMP1, MMP3, and MMP13, which are strongly related to OA development, increased in LPS-stimulated chondrocytes. However, AKBA treatment significantly reversed the LPS-induced upregulation of MMPs at protein (Fig. 3(I), (L)-(M)) and mRNA (Fig. 3(Q)-(S)) levels.

Overall, these results demonstrated that AKBA could efficiently reverse ECM and maintain the phenotype in LPS-induced rat articular chondrocytes.

\subsection{AKBA treatment inhibited NF-KB activation in vitro and in vivo}


We detected the expression of pivotal markers involved in related signaling pathways to examine the underlying molecular mechanisms of AKBA intervention. IHC staining of p65, IKKa, and IKBa was performed to confirm that AKBA treatment could suppress surgery-induced inflammation and ECM degradation via NF-KB signaling pathway in rat knees. There was a high expression of p65 and IKKa in the model group and intense IKBa staining in AKBA-treated rats, compared with the low expression observed in vehicle rats (Fig. 4(A)). Semi-quantitative analysis indicated that compared with controls, the removal of ligaments and meniscus increased the expression of p65 and IKKa but reduced IKBa expression, respectively (Fig. 4(B)-(D)). AKBA intervention corrected the effects of surgical stimulation. These data showed that AKBA alleviated surgery-induced inflammation and ECM degradation via suppression of the NF-KB signaling pathway in vivo.

Among the three classic $1 \mathrm{kB}$ proteins, $\mathrm{IKBa}$ is the strongest negative feedback factor in the activation of $\mathrm{NF}-\mathrm{KB}$, which ensures the rapid occurrence and shutdown of NF-KB activation. The classical NF-KB signaling pathway was activated and IKBa was rapidly degraded upon LPS induction (Fig. 5(A)-(C)). Interestingly, after AKBA treatment, the degradation of IKBa was restored. Western blot and qRT-PCR analyses demonstrated that LPS activated the levels of p65. However, the p65 level was markedly decreased in AKBA-treated chondrocytes compared to LPS-treated cells only. Western blot analysis of $p$ $I K K a / \beta$, IKKa, IKK $\beta, p-p 65, p 65, p-I K B a$, and IKBa showed that AKBA intervention affected the ratio of $p$ $\mathrm{IKKa} / \beta$ to IKKa plus IKK $\beta$, $p-p 65$ to $p 65$ and $p-I K B a$ to $I K B a$ induced by LPS, respectively. These results showed that the protective effects of AKBA may be mediated by repressing the NF-KB signaling pathway.

3-O-C12-HSL is an acyl-homoserine lactone (AHL), an activator of NF-KB. We first confirmed that 3-O-C12HSL could promote inflammation and ECM degradation. Western blotting (Fig. S4) showed a drastic increase in the expression of inflammatory factors and MMPs after 3-0-C12-HSL treatment. As expected, the expression of aggrecan and collagen II were drastically inhibited in 3-0-C12-HSL-treated chondrocytes compared to controls (Fig. S4(B), (F), and(G)). Moreover, we observed the effects of 3-O-C12-HSL on AKBA-treated chondrocytes. Medicare, $5 \mu \mathrm{M}$ of 3-0-C12-HSL elevated levels of inflammatory factors and MMPs, and decreased protein and mRNA levels of aggrecan and collagen II at the same time(Fig. S5).

Strikingly, treatment with $5 \mu \mathrm{M}$ of 3-0-C12-HSL (contained $1 \mu \mathrm{g} / \mathrm{ml}$ LPS and $8 \mu \mathrm{M}$ AKBA) reversed the AKBA-mediated rescue of the LPS-induced activation of the NF-kB signaling pathway. The results indicated that 3-O-C12-HSL activated protein expression of p65 and IKBa (Fig. S6(A)-(C)). IF analysis also indicated that AKBA intervention promoted the inhibition of $\mathrm{p} 65$ and decreased its nuclear translocation (Fig. S6(D)). Nevertheless, these effects were suppressed after the intervention of 3-0-C12-HSL (Fig. S6(F)). Overall, 3-O-C12-HSL severely inhibited the protective effect of AKBA on NF-KB inhibition. These findings denoted that AKBA intervention resolved the activation of the LPS-induced NF-KB signaling pathway, while 3-0-C12-HSL treatment reversed the positive effect of AKBA in LPS-induced inflammation and ECM degradation.

\section{Discussion}


Mounting studies have identified that a combination of biological and mechanical factors disrupts the synthesis and degradation balance of chondrocytes, ECM, and subchondral bone, causing cartilage degeneration, fibrosis, and subchondral callus.(Xia et al., 2014) (Lieberthal et al., 2015) (Carballo et al., 2017) (Y. Wang et al., 2017) (Trachana et al., 2019) Mechanical stimulation leads to metabolic changes, which are characterized by the release of inflammatory mediators and MMPs and the degradation of ECM.(Mehana et al., 2019) (Yang et al., 2020) (Philpott et al., 2017) (Rahmati et al., 2017) Under normal circumstances, anabolism and catabolism of ECM are in a dynamic equilibrium, which is essential for a healthy joint.(Rahmati et al., 2017) Once the balance is disrupted, it leads to irreparable joint cartilage damage and OA symptoms. Alleviating the inflammatory mediators and ECM degradation is a key player in the prevention versus treatment of OA. Despite some breakthroughs made in illuminating the pathogenesis of $O A$, there are no disease-modifying drugs with evidently validated therapeutic effects to modify the progression of OA at present.(Quicke et al., 2022) (Jiang, 2022) (Lieberthal et al., 2015)

Natural products could treat various human diseases, including OA, after their widespread use, half of which are likely extracted from plants.(Zhou et al., 2019) (Wu et al., 2019) (Z. Wang et al., 2017) (Y. Hu et al., 2019) (H. Hu et al., 2018) AKBA, a pharmacologically active pentacyclic triterpenes compound from Boswellia serrate extract, has been reported previously to alleviate host inflammatory response, mitigating inflammation-induced damage to diabetes, bronchial and nervous tissue.(Meka et al., 2017) (Takada et al., 2006) (Ding et al., 2016) (Sayed et al., 2018) In addition, it has been reported that AKBA has anti-osteogenic inhibition, anti-ulcer, anti-asthma, anti-oxidative stress, anti-tumor, and lipid regulatory effects.(Tambe et al., 2019) (Rajabian et al., 2020) (Li et al., 2018) AKBA inhibited inflammation through NF-KB-regulated gene expression. We concluded that AKBA reversed the imbalance of LPS-induced inflammation and ECM metabolism by inhibiting the NF-KB pathway. Additionally, we demonstrated that AKBA intervention alleviated the progress of OA in a rat Hulth-Telhag OA model for the first time.

Several studies have reported that the NF-KB signaling pathway plays an important role in the pathophysiological process of $O A$ and is activated by the inflammation of $O A$, as such, the upstream regulators, co-factors, and downstream effectors of NF-KB have become promising targets of OA and rheumatoid arthritis therapies.(Lepetsos et al., 2019) (Napetschnig \& Wu, 2013) (Jimi et al., 2019) Based on current studies, NF-KB plays a vital part in inducing various inflammatory-related factors, including IL$1 \beta$, IL-6, TNF-a, and MMP proteins, which can stimulate chondrocyte catabolism.(Herrero-Beaumont et al., 2019) (Catrysse \& van Loo, 2017) (Khan et al., 2017) NF-KB is also related to ECM degradation. As the two most abundant components in the ECM, aggrecan and col II are degraded by MMPs, whose increased expression is regulated by NF-KB.(Hussain et al., 2018) (Luo et al., 2019) Furthermore, NF-kB can phosphorylate and degrade IKB protein when chondrocytes are stimulated by various stimuli, causing the transport of NF-KB p65 to the nucleus, where p65 facilitates OA-related gene and protein expression. Our study suggested that AKBA treatment inhibited the overexpression of IL-1 $\beta$, IL-6, and TNF-a using Western blot and qRT-PCR. Further, AKBA treatment reversed the degradation of aggrecan and col II and the upregulation of MMPs. The results also suggested that AKBA treatment could inhibit nuclear translocation of p-p65 by Western blot and IF analyses. Taken together, these results showed that AKBA 
treatment plays a positive role in anti-inflammation and ECM degradation by inactivating the NF-KB signaling pathway (Fig. 6).

3-0-C12-HSL, one of the AHLs frequently identified in extracts of respiratory secretions from cystic fibrosis patients infected with Pseudomonas aeruginosa and Burkholderia cepacia complex species and acts as an activator of NF-kB, was used to verify the role of the NF-kB pathway.(Henke \& Bassler, 2004) (Chambers et al., 2005) (Smith et al., 2001) IF assay indicated that AKBA treatment depressed nuclear translocation of $\mathrm{p} 65$ after LPS-induced cytoplasmic localization. However, 3-0-C12-HSL treatment reversed the effects of AKBA on the inhibition of p65 in chondrocytes. Overall, our results demonstrated that AKBA intervention restrained LPS-induced inflammation and ECM degradation via suppression of the NF-KB signaling pathway.

\section{Conclusion}

In this integrative study, we found that AKBA alleviated inflammation and ECM degradation, which may be related to the regulation of NF-KB. However, the effects of other related signaling pathways have not been ruled out and should be investigated in subsequent experiments. Besides, we found that osteoclast was more active in the vehicle group than in controls, which was consistent with micro-CT findings. These results suggest that AKBA may be a potential drug target to prevent and treat OA by enhancing anti-inflammatory and inhibiting ECM degradation.

\section{Abbreviations}

OA: osteoarthritis; AKBA: acetyl-11-keto- $\beta$-boswellic acid; KBA: 11-keto- $\beta$-boswellic acid; MMP: matrix metalloproteinase; ECM: extracellular matrix; SD: Sprague Dawley; Tb. BMD: Bone trabecular bone density; BV/TV: bone volume fraction; Tb. N: number of trabecular bone; Tb. Th: thickness of trabecular bone; Tb. Sp: trabecular bone separation; Conn. D: connection density; H\&E: hematoxylin and eosin; IHC: immunohistochemistry; IF: immunofluorescence; OcS/BS: the proportion of osteoclast surface; LPS: lipopolysaccharide; NF-KB: nuclear factor kappa B; NSAIDs: nonsteroidal anti-inflammatory drugs; IL: interleukin; TNF: tumor necrosis factor; TRAP: tartrate-resistant acid phosphatase; OARSI: International Academy of Osteoarthritis Research; RT: room temperature; FBS: fetal bovine serum; PBS: phosphate buffer saline; CCK-8: cell counting Kit-8; RIPA: radioimmunoprecipitation assay; PVDF: polyvinylidene difluoride; qRT-PCR: quantitative real-time PCR; DAPI:2-(4-Amidinophenyl)-6-indolecarbamidine dihydrochloride.

\section{Declarations}

\section{Ethics approval and consent to participate}


Animal welfare and experimental procedures were carried out in accordance with the Declaration of Helsinke, and were approved by the Ethics Committee of the First Affiliated Hospital of Soochow University.

\section{Consent for publication}

We declare that the Publisher has the Author's permission to publish the relevant contribution.

\section{Availability of data and materials}

Data available within the article or its supplementary materials.

\section{Competing interests}

The authors declare no competing confct of interests.

\section{Fundings}

Financial supports from National Natural Science Foundation of China (No. 82072425, 81873991 and 81672238); Natural Science Foundation of Jiangsu Province (No. BK20180001 and BK20211083); Jiangsu Commission of Health (No. H2018027); Jiangsu social development project (No. BE2021673 and BE2022737); Graduate Research and Practice innovation Plan of Graduate Education Innovation Project in Jiangsu Province (KYCX21_1578); Suzhou Jiangsu Commission of Health (No. SYS2019100 and SYS2019101), Suzhou Health Committee gusu Health Personnel Training project (GSWS2020078); Project of Suzhou Sports Research Bureau (No. TY2019-204), and Special Project of Diagnosis and Treatment Technology for Key Clinical Diseases in Suzhou (LCZX202003).

\section{Authors' contributions}

Jing Zhou: writing - review \& editing, data curation, methodology. Zeyu Han: data curation, methodology. Xueyan Li: data curation, methodology. Zhiqiang Shao: data curation, methodology. Yinhua Qian: data curation, methodology. Lang Bai: data curation, software. Qibin Han: investigation, validation. Xiaoyu Zhang: investigation, validation. Qi Chen: methodology. Maofeng Gao: formal analysis, methodology. Yi Xue: formal analysis, methodology. Dechun Geng: funding acquisition, resources. Xing Yang: supervision, project administration. Yuefeng Hao: conceptualization, supervision.

\section{Acknowledgements}

We would like to thank the Institute of Orthopedics, Soochow University limited for their technical support.

\section{References}

1. Carballo, C. B., Nakagawa, Y., Sekiya, I., \& Rodeo, S. A. (2017). Basic Science of Articular Cartilage. Clinics in Sports Medicine, 36(3), 413-425. https://doi.org/10.1016/j.csm.2017.02.001 
2. Catrysse, L., \& van Loo, G. (2017). Inflammation and the Metabolic Syndrome: The Tissue-Specific Functions of NF-KB. Trends in Cell Biology, 27(6), 417-429.

https://doi.org/10.1016/j.tcb.2017.01.006

3. Chambers, C. E., Visser, M. B., Schwab, U., \& Sokol, P. A. (2005). Identification of N-acylhomoserine lactones in mucopurulent respiratory secretions from cystic fibrosis patients. FEMS Microbiology Letters, 244(2), 297-304. https://doi.org/10.1016/j.femsle.2005.01.055

4. Ding, Y., Qiao, Y., Wang, M., Zhang, H., Li, L., Zhang, Y., Ge, J., Song, Y., Li, Y., \& Wen, A. (2016). Enhanced Neuroprotection of Acetyl-11-Keto- $\beta$-Boswellic Acid (AKBA)-Loaded O-Carboxymethyl Chitosan Nanoparticles Through Antioxidant and Anti-Inflammatory Pathways. Molecular Neurobiology, 53(6), 3842-3853. https://doi.org/10.1007/s12035-015-9333-9

5. Glyn-Jones, S., Palmer, A. J. R., Agricola, R., Price, A. J., Vincent, T. L., Weinans, H., \& Carr, A. J. (2015). Osteoarthritis. Lancet (London, England), 386(9991), 376-387. https://doi.org/10.1016/S01406736(14)60802-3

6. Henke, J. M., \& Bassler, B. L. (2004). Bacterial social engagements. Trends in Cell Biology, 14(11), 648-656. https://doi.org/10.1016/j.tcb.2004.09.012

7. Herrero-Beaumont, G., Pérez-Baos, S., Sánchez-Pernaute, O., Roman-Blas, J. A., Lamuedra, A., \& Largo, R. (2019). Targeting chronic innate inflammatory pathways, the main road to prevention of osteoarthritis progression. Biochemical Pharmacology, 165, 24-32.

https://doi.org/10.1016/j.bcp.2019.02.030

8. Hu, H., Li, Y., Xin, Z., \& Zhanga, X. (2018). Ginkgolide B exerts anti-inflammatory and chondroprotective activity in LPS-induced chondrocytes. Advances in Clinical and Experimental Medicine: Official Organ Wroclaw Medical University, 27(7), 913-920.

https://doi.org/10.17219/acem/70414

9. Hu, Y., Gui, Z., Zhou, Y., Xia, L., Lin, K., \& Xu, Y. (2019). Quercetin alleviates rat osteoarthritis by inhibiting inflammation and apoptosis of chondrocytes, modulating synovial macrophages polarization to M2 macrophages. Free Radical Biology \& Medicine, 145, 146-160. https://doi.org/10.1016/j.freeradbiomed.2019.09.024

10. Hussain, S., Sun, M., Min, Z., Guo, Y., Xu, J., Mushtaq, N., Heng, L., Huang, H., Zhao, Y., Yuan, Y., Hussain, N., Zhang, F., Han, Y., Xu, P., Sun, J., \& Lu, S. (2018). Down-regulated in OA cartilage, SFMBT2 contributes to NF-KB-mediated ECM degradation. Journal of Cellular and Molecular Medicine, 22(11), 5753-5758. https://doi.org/10.1111/jcmm.13826

11. Jiang, Y. (2022). Osteoarthritis year in review 2021: Biology. Osteoarthritis and Cartilage, 30(2), 207215. https://doi.org/10.1016/j.joca.2021.11.009

12. Jimi, E., Fei, H., \& Nakatomi, C. (2019). NF-kB Signaling Regulates Physiological and Pathological Chondrogenesis. International Journal of Molecular Sciences, 20(24), E6275. https://doi.org/10.3390/ijms20246275

13. Khan, N. M., Haseeb, A., Ansari, M. Y., Devarapalli, P., Haynie, S., \& Haqqi, T. M. (2017). Wogonin, a plant derived small molecule, exerts potent anti-inflammatory and chondroprotective effects through 
the activation of ROS/ERK/Nrf2 signaling pathways in human Osteoarthritis chondrocytes. Free Radical Biology \& Medicine, 106, 288-301. https://doi.org/10.1016/j.freeradbiomed.2017.02.041

14. Lepetsos, P., Papavassiliou, K. A., \& Papavassiliou, A. G. (2019). Redox and NF-KB signaling in osteoarthritis. Free Radical Biology \& Medicine, 132, 90-100. https://doi.org/10.1016/j.freeradbiomed.2018.09.025

15. Li, W., Liu, J., Fu, W., Zheng, X., Ren, L., Liu, S., Wang, J., Ji, T., \& Du, G. (2018). 3-0-acetyl-11-keto- $\beta-$ boswellic acid exerts anti-tumor effects in glioblastoma by arresting cell cycle at $\mathrm{G} 2 / \mathrm{M}$ phase. Journal of Experimental \& Clinical Cancer Research: CR, 37(1), 132. https://doi.org/10.1186/s13046018-0805-4

16. Lieberthal, J., Sambamurthy, N., \& Scanzello, C. R. (2015). Inflammation in joint injury and posttraumatic osteoarthritis. Osteoarthritis and Cartilage, 23(11), 1825-1834. https://doi.org/10.1016/j.joca.2015.08.015

17. Loeser, R. F., Collins, J. A., \& Diekman, B. O. (2016). Ageing and the pathogenesis of osteoarthritis. Nature Reviews. Rheumatology, 12(7), 412-420. https://doi.org/10.1038/nrrheum.2016.65

18. Luo, Z., Zheng, B., Jiang, B., Xue, X., Xue, E., \& Zhou, Y. (2019). Peiminine inhibits the IL-1 $\beta$ induced inflammatory response in mouse articular chondrocytes and ameliorates murine osteoarthritis. Food \& Function, 10(4), 2198-2208. https://doi.org/10.1039/c9fo00307j

19. Mehana, E.-S. E., Khafaga, A. F., \& El-Blehi, S. S. (2019). The role of matrix metalloproteinases in osteoarthritis pathogenesis: An updated review. Life Sciences, 234, 116786. https://doi.org/10.1016/j.lfs.2019.116786

20. Meka, B., Ravada, S. R., Murali Krishna Kumar, M., Purna Nagasree, K., \& Golakoti, T. (2017). Synthesis of new analogs of AKBA and evaluation of their anti-inflammatory activities. Bioorganic \& Medicinal Chemistry, 25(4), 1374-1388. https://doi.org/10.1016/j.bmc.2016.12.045

21. Miscioscia, E., Shmalberg, J., \& Scott, K. C. (2019). Measurement of 3-acetyl-11-keto-beta-boswellic acid and 11-keto-beta-boswellic acid in Boswellia serrata Supplements Administered to Dogs. BMC Veterinary Research, 15(1), 270. https://doi.org/10.1186/s12917-019-2021-7

22. Napetschnig, J., \& Wu, H. (2013). Molecular basis of NF-KB signaling. Annual Review of Biophysics, 42, 443-468. https://doi.org/10.1146/annurev-biophys-083012-130338

23. Ning, Z., Wang, C., Liu, Y., Song, Z., Ma, X., Liang, D., Liu, Z., \& Lu, A. (2018). Integrating Strategies of Herbal Metabolomics, Network Pharmacology, and Experiment Validation to Investigate Frankincense Processing Effects. Frontiers in Pharmacology, 9, 1482. https://doi.org/10.3389/fphar.2018.01482

24. Philpott, H. T., O’Brien, M., \& McDougall, J. J. (2017). Attenuation of early phase inflammation by cannabidiol prevents pain and nerve damage in rat osteoarthritis. Pain, 158(12), 2442-2451. https://doi.org/10.1097/j.pain.0000000000001052

25. Quicke, J. G., Conaghan, P. G., Corp, N., \& Peat, G. (2022). Osteoarthritis year in review 2021: Epidemiology \& therapy. Osteoarthritis and Cartilage, 30(2), 196-206. https://doi.org/10.1016/j.joca.2021.10.003 
26. Rahmati, M., Nalesso, G., Mobasheri, A., \& Mozafari, M. (2017). Aging and osteoarthritis: Central role of the extracellular matrix. Ageing Research Reviews, 40, 20-30.

https://doi.org/10.1016/j.arr.2017.07.004

27. Rajabian, A., Sadeghnia, H. R., Hosseini, A., Mousavi, S. H., \& Boroushaki, M. T. (2020). 3-Acetyl-11keto- $\beta$-boswellic acid attenuated oxidative glutamate toxicity in neuron-like cell lines by apoptosis inhibition. Journal of Cellular Biochemistry, 121(2), 1778-1789. https://doi.org/10.1002/jcb.29413

28. Reyes, C., Leyland, K. M., Peat, G., Cooper, C., Arden, N. K., \& Prieto-Alhambra, D. (2016). Association Between Overweight and Obesity and Risk of Clinically Diagnosed Knee, Hip, and Hand Osteoarthritis: A Population-Based Cohort Study. Arthritis \& Rheumatology (Hoboken, N.J.), 68(8), 1869-1875. https://doi.org/10.1002/art.39707

29. Roy, N. K., Parama, D., Banik, K., Bordoloi, D., Devi, A. K., Thakur, K. K., Padmavathi, G., Shakibaei, M., Fan, L., Sethi, G., \& Kunnumakkara, A. B. (2019). An Update on Pharmacological Potential of Boswellic Acids against Chronic Diseases. International Journal of Molecular Sciences, 20(17), E4101. https://doi.org/10.3390/ijms20174101

30. Sayed, A. S., Gomaa, I. E. O., Bader, M., \& El Sayed, N. S. E. D. (2018). Role of 3-Acetyl-11-Keto-BetaBoswellic Acid in Counteracting LPS-Induced Neuroinflammation via Modulation of miRNA-155. Molecular Neurobiology, 55(7), 5798-5808. https://doi.org/10.1007/s12035-017-0801-2

31. Sengupta, K., Alluri, K. V., Satish, A. R., Mishra, S., Golakoti, T., Sarma, K. V., Dey, D., \& Raychaudhuri, S. P. (2008). A double blind, randomized, placebo controlled study of the efficacy and safety of 5-Loxin for treatment of osteoarthritis of the knee. Arthritis Research \& Therapy, 10(4), R85. https://doi.org/10.1186/ar2461

32. Smith, R. S., Fedyk, E. R., Springer, T. A., Mukaida, N., Iglewski, B. H., \& Phipps, R. P. (2001). IL-8 production in human lung fibroblasts and epithelial cells activated by the Pseudomonas autoinducer $\mathrm{N}-3-0 x o d o d e c a n o y l$ homoserine lactone is transcriptionally regulated by NF-kappa B and activator protein-2. Journal of Immunology (Baltimore, Md.: 1950), 167(1), 366-374.

https://doi.org/10.4049/jimmunol.167.1.366

33. Takada, Y., Ichikawa, H., Badmaev, V., \& Aggarwal, B. B. (2006). Acetyl-11-keto-beta-boswellic acid potentiates apoptosis, inhibits invasion, and abolishes osteoclastogenesis by suppressing NF-kappa B and NF-kappa B-regulated gene expression. Journal of Immunology (Baltimore, Md.: 1950), 176(5), 3127-3140. https://doi.org/10.4049/jimmunol.176.5.3127

34. Tambe, A., Mokashi, P., \& Pandita, N. (2019). Ex-vivo intestinal absorption study of boswellic acid, cyclodextrin complexes and poloxamer solid dispersions using everted gut sac technique. Journal of Pharmaceutical and Biomedical Analysis, 167, 66-73. https://doi.org/10.1016/j.jpba.2018.12.018

35. Trachana, V., Mourmoura, E., Papathanasiou, I., \& Tsezou, A. (2019). Understanding the role of chondrocytes in osteoarthritis: Utilizing proteomics. Expert Review of Proteomics, 16(3), 201-213. https://doi.org/10.1080/14789450.2019.1571918

36. Wang, Y., Yu, D., Liu, Z., Zhou, F., Dai, J., Wu, B., Zhou, J., Heng, B. C., Zou, X. H., Ouyang, H., \& Liu, H. (2017). Exosomes from embryonic mesenchymal stem cells alleviate osteoarthritis through 
balancing synthesis and degradation of cartilage extracellular matrix. Stem Cell Research \& Therapy, 8(1), 189. https://doi.org/10.1186/s13287-017-0632-0

37. Wang, Z., Huang, J., Zhou, S., Luo, F., Xu, W., Wang, Q., Tan, Q., Chen, L., Wang, J., Chen, H., Chen, L., Xie, Y., \& Du, X. (2017). Anemonin attenuates osteoarthritis progression through inhibiting the activation of IL-1 $\beta / N F-k B$ pathway. Journal of Cellular and Molecular Medicine, 21(12), 3231-3243. https://doi.org/10.1111/jcmm.13227

38. Wu, Y., Lin, Z., Yan, Z., Wang, Z., Fu, X., \& Yu, K. (2019). Sinomenine contributes to the inhibition of the inflammatory response and the improvement of osteoarthritis in mouse-cartilage cells by acting on the Nrf2/HO-1 and NF-kB signaling pathways. International Immunopharmacology, 75, 105715. https://doi.org/10.1016/j.intimp.2019.105715

39. Xia, B., Di Chen, null, Zhang, J., Hu, S., Jin, H., \& Tong, P. (2014). Osteoarthritis pathogenesis: A review of molecular mechanisms. Calcified Tissue International, 95(6), 495-505. https://doi.org/10.1007/s00223-014-9917-9

40. Xiong, L., Liu, Y., Zhu, F., Lin, J., Wen, D., Wang, Z., Bai, J., Ge, G., Xu, C., Gu, Y., Xu, Y., Zhou, J., \& Geng, D. (2019). Acetyl-11-keto- $\beta$-boswellic acid attenuates titanium particle-induced osteogenic inhibition via activation of the GSK-3 $\beta / \beta$-catenin signaling pathway. Theranostics, $9(24), 7140-7155$. https://doi.org/10.7150/thno.35988

41. Yang, Y., Wang, Y., Kong, Y., Zhang, X., Zhang, H., Feng, X., Wang, Z., Gao, P., Yan, M., Bai, L., \& Li, F. (2020). Moderate Mechanical Stimulation Protects Rats against Osteoarthritis through the Regulation of TRAIL via the NF-KB/NLRP3 Pathway. Oxidative Medicine and Cellular Longevity, 2020, 6196398. https://doi.org/10.1155/2020/6196398

42. Zeng, C., Wei, J., Persson, M. S. M., Sarmanova, A., Doherty, M., Xie, D., Wang, Y., Li, X., Li, J., Long, H., Lei, G., \& Zhang, W. (2018). Relative efficacy and safety of topical non-steroidal anti-inflammatory drugs for osteoarthritis: A systematic review and network meta-analysis of randomised controlled trials and observational studies. British Journal of Sports Medicine, 52(10), 642-650. https://doi.org/10.1136/bjsports-2017-098043

43. Zhou, F., Mei, J., Han, X., Li, H., Yang, S., Wang, M., Chu, L., Qiao, H., \& Tang, T. (2019). Kinsenoside attenuates osteoarthritis by repolarizing macrophages through inactivating NF-KB/MAPK signaling and protecting chondrocytes. Acta Pharmaceutica Sinica. B, 9(5), 973-985. https://doi.org/10.1016/j.apsb.2019.01.015

\section{Figures}


A

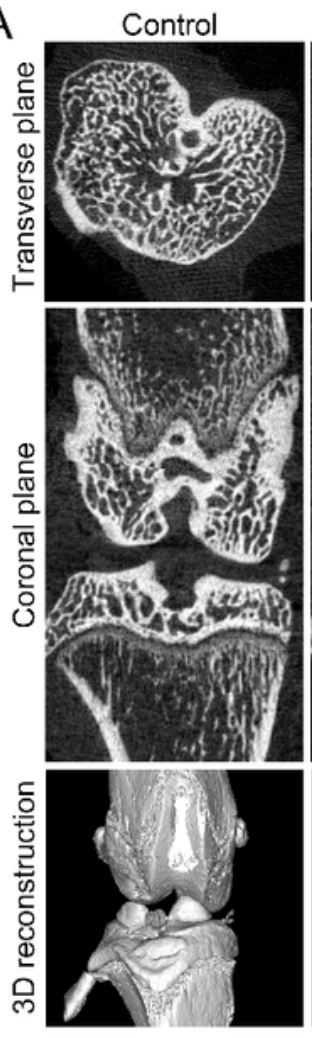

$\mathrm{H}$

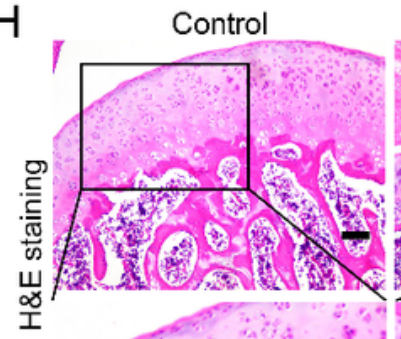

Vehicle
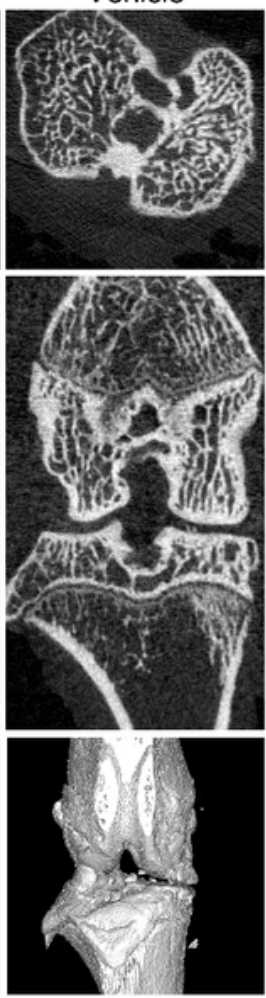

Low-AKBA
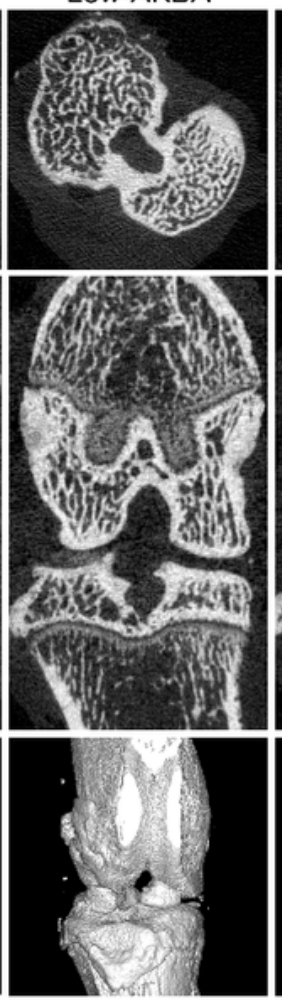

High-AKBA
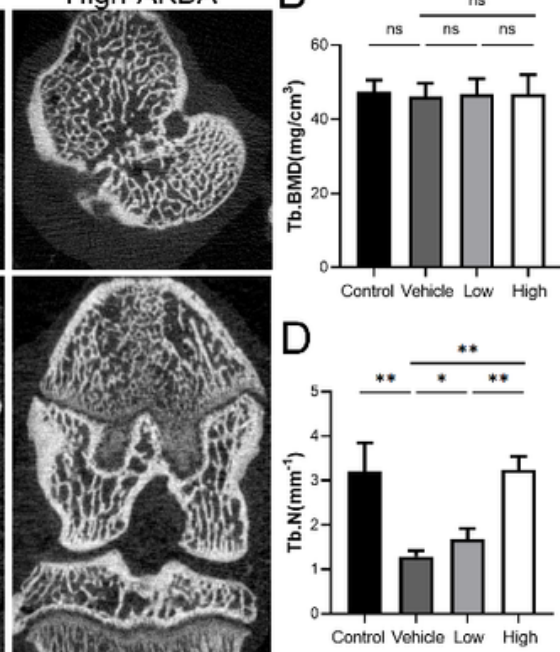

F

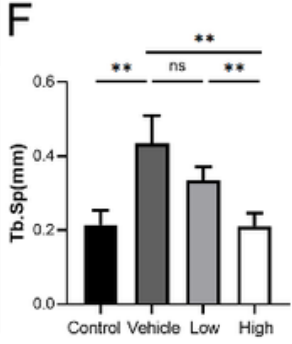

High-AKBA
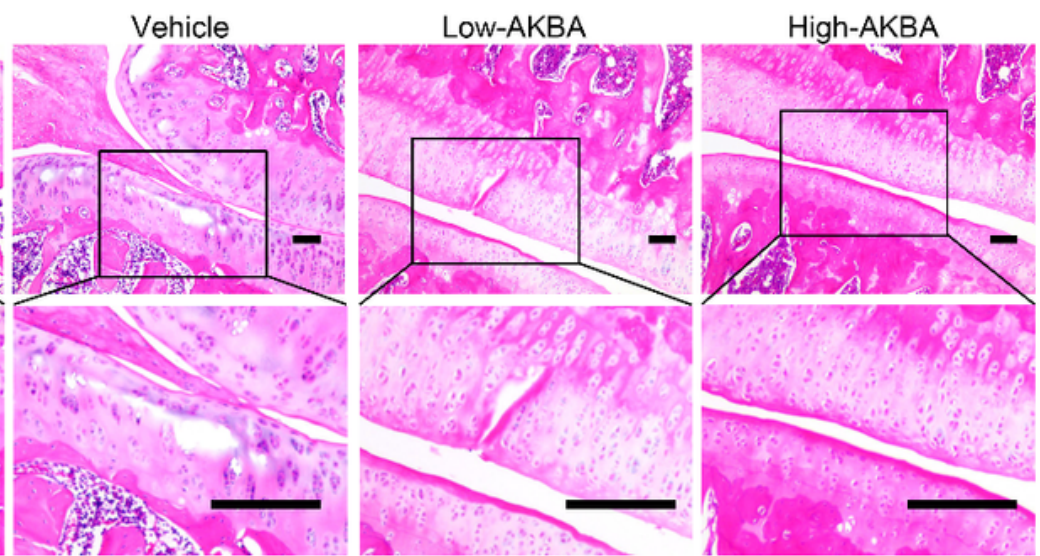

C

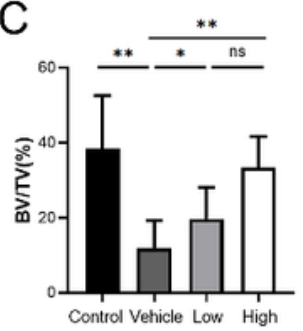

E

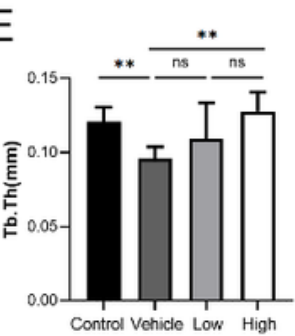

G

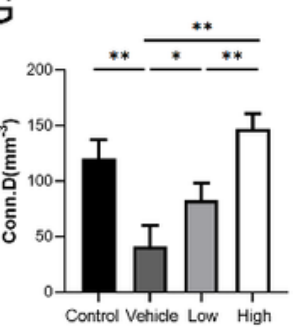

I
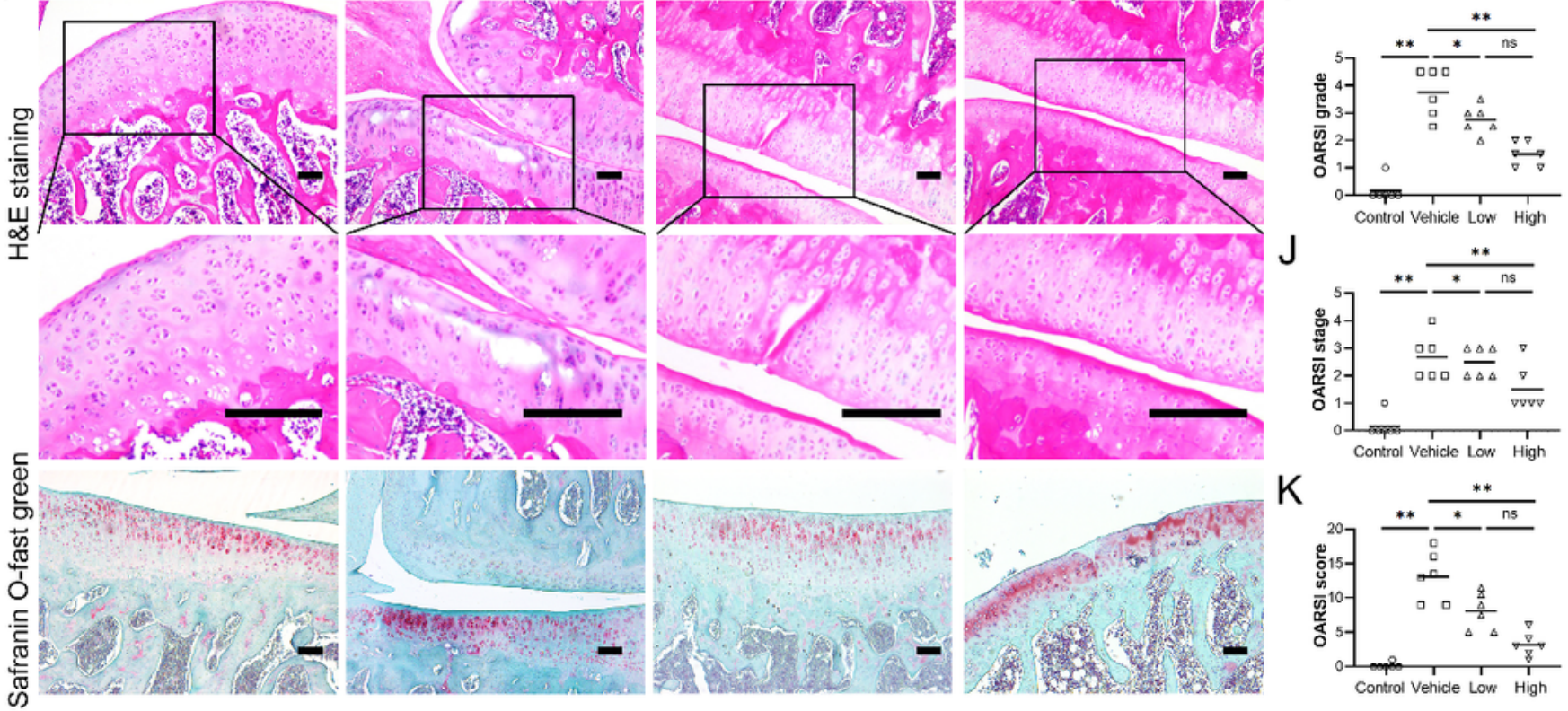

Figure 1

AKBA treatment attenuated surgery-induced bone loss and increased bone mass in a rat Hulth-Telhag model. (a) micro-CT reconstruction images from different experimental groups, (b-g) Bone trabecular bone density (Tb. BMD, $\mathrm{mg} / \mathrm{cm}^{3}$ ), bone volume fraction (BV/TV, \%), number of trabecular bone (Tb. $\mathrm{N}$, $\mathrm{mm}^{-1}$ ), thickness of trabecular bone (Tb. Th, $\left.\mu \mathrm{m}\right)$, trabecular bone separation (Tb. Sp, $\mathrm{mm}$ ), and connection density (Conn. D, mm $\left.{ }^{-1}\right)$, (h) H\&E staining and safranin O-fast green staining, (i-k) OARSI 
grade, OARSI stage, and OARSI score. Both low-AKBA ( $2 \mathrm{mg} / \mathrm{kg}$ every other day) group and high-AKBA (8 $\mathrm{mg} / \mathrm{kg}$ every other day) group received surgery intervention. $\mathrm{n}=6$ per group. Scale bar $=100 \mu \mathrm{m}$. Data are presented as means $\pm S D$. ${ }^{*} P<0.05$ and ${ }^{*} * P<0.01$, vs. vehicle group.

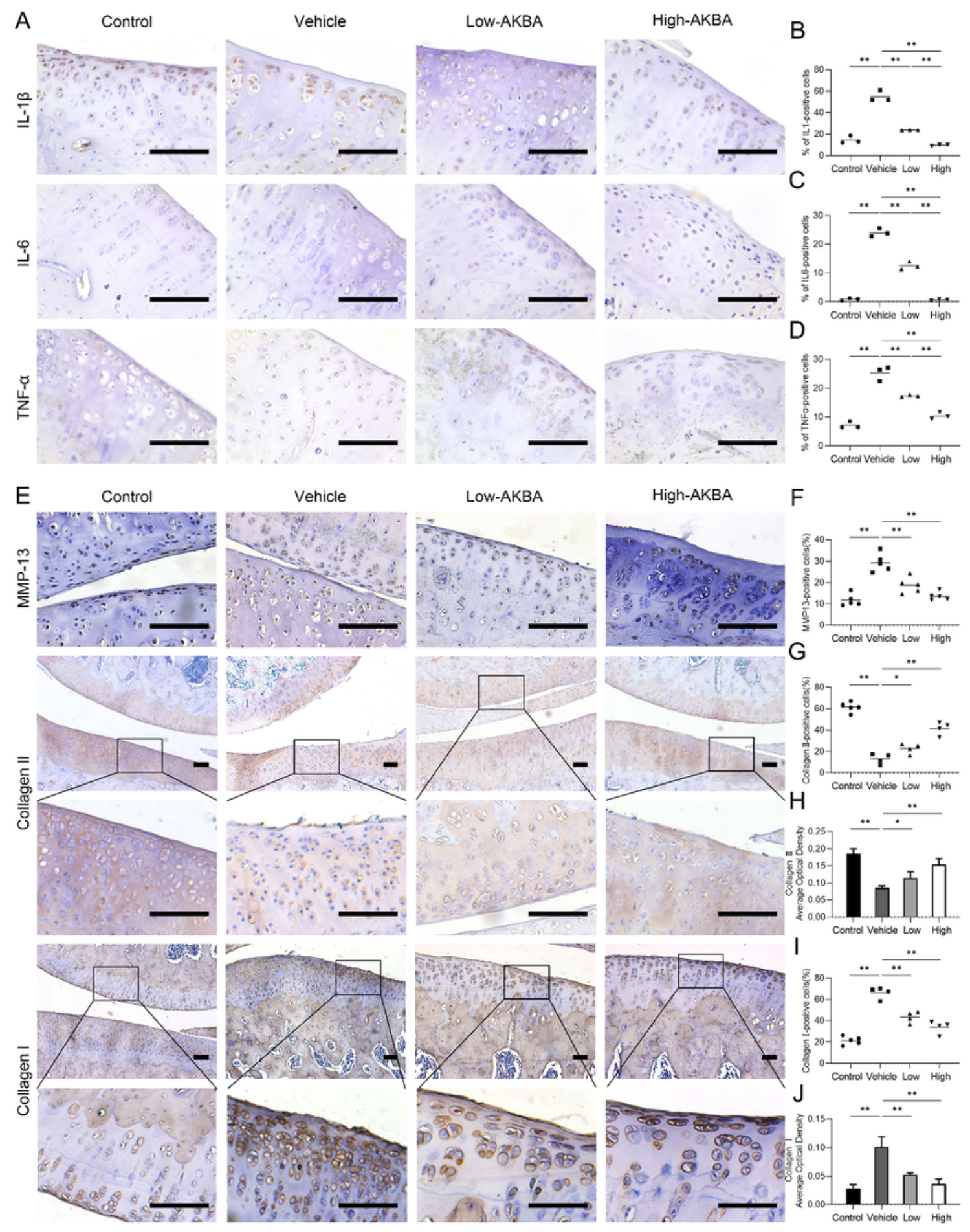

Figure 2 
AKBA intervention attenuated inflammation and ECM degradation in a rat Hulth-Telhag OA model. (a) IHC for IL-1 $\beta$, IL-6, and TNF- $a$. (b-d) The percentage of cells positive for IL-1 $\beta$, IL-6, and TNF-a. (e) IHC for MMP-13, col II, and col I. (f, $g$, and i) The percentage of cells positive for MMP-13, col II, and col I. (h, j) Average optical density for col II and col I. Both low-AKBA ( $2 \mathrm{mg} / \mathrm{kg}$ every other day) group and highAKBA ( $8 \mathrm{mg} / \mathrm{kg}$ every other day) group received surgery intervention. $\mathrm{n}=6$ per group. Scale bar $=100 \mu \mathrm{m}$. Data are presented as means \pm SD. $* P<0.05$ and $* * P<0.01$, vs. vehicle group.
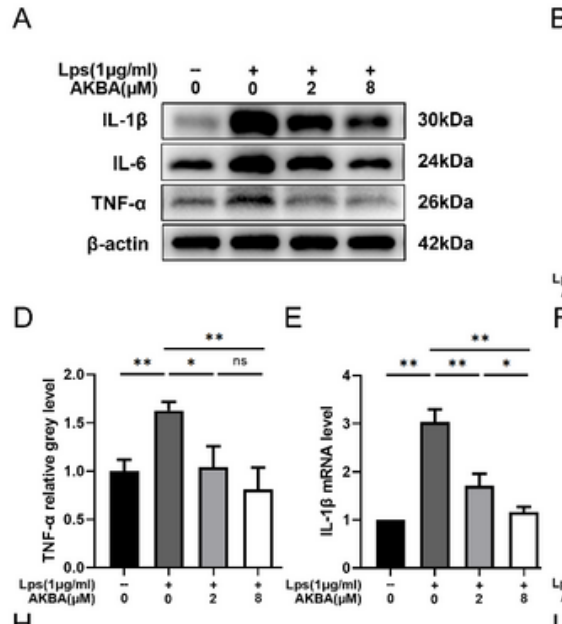

$\mathrm{H}$

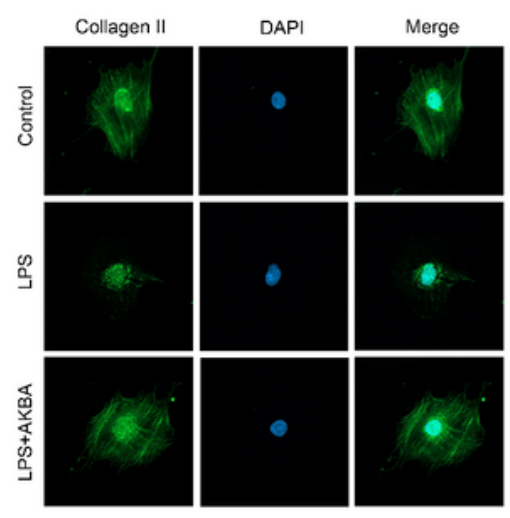

L

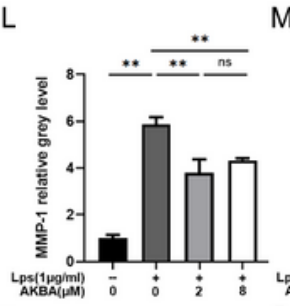

M
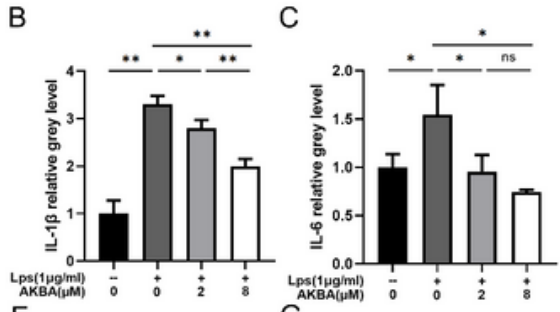

$\mathrm{F}$
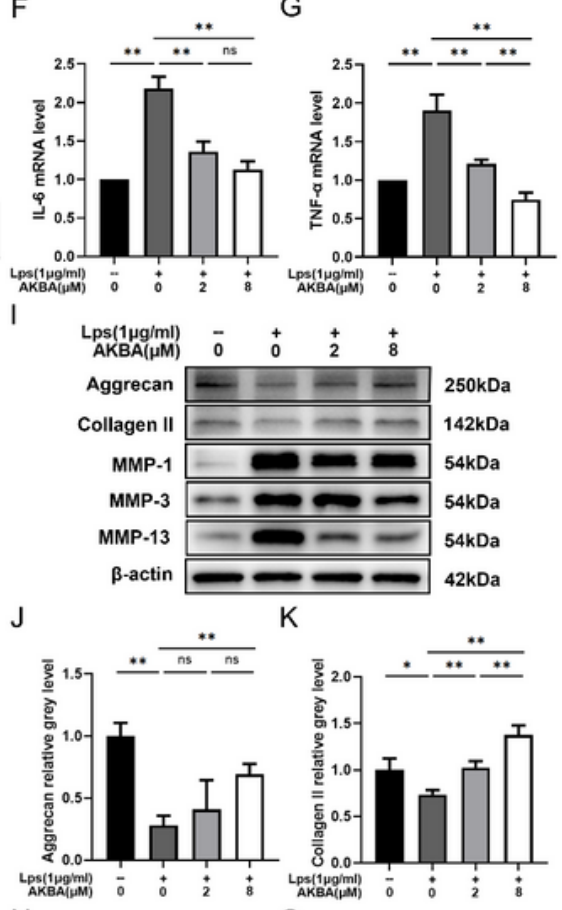

N
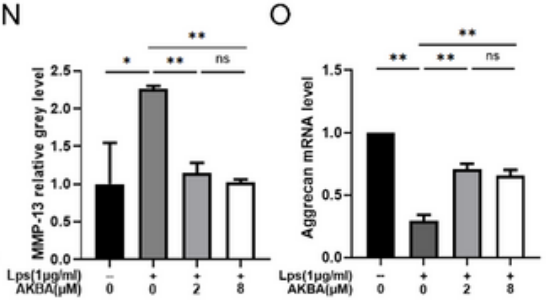

$\mathrm{R}$

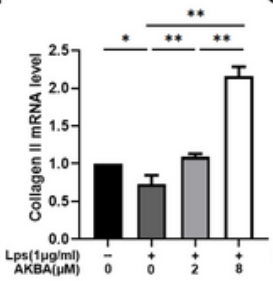

Q
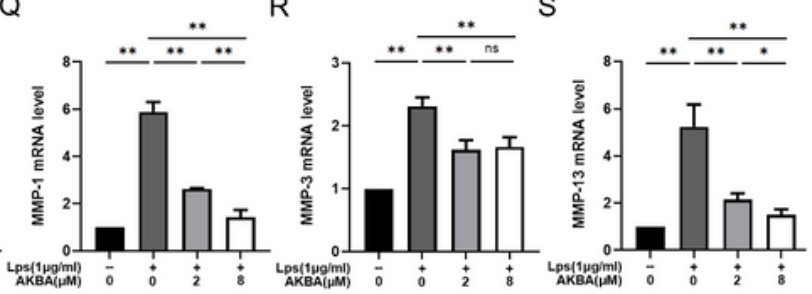
Figure 3

AKBA intervention attenuated inflammation and ECM degradation in LPS-induced rat chondrocytes. (a-d) IL-1 $\beta$, IL-6, and TNF-a protein expression levels. (e-g) IL-1 $\beta$, IL-6, and TNF-a mRNA expression levels. (h) Cellular immunofluorescence of col II in chondrocytes. (i-n) Agg, col II, MMP-1, MMP-3, and MMP-13 protein expression levels. (o-s) Agg, col II, MMP-1, MMP-3, and MMP-13 mRNA expression levels. Relative grey levels were quantified using Image J. Both low-AKBA $(2 \mu \mathrm{M})$ group and high-AKBA $(8 \mu \mathrm{M})$ group contained $1 \mu \mathrm{g} / \mathrm{ml}$ LPS. Dates are presented as means \pm SD of six duplicate experiments. ${ }^{*} P<0.05$, $\star * P<0.01$.
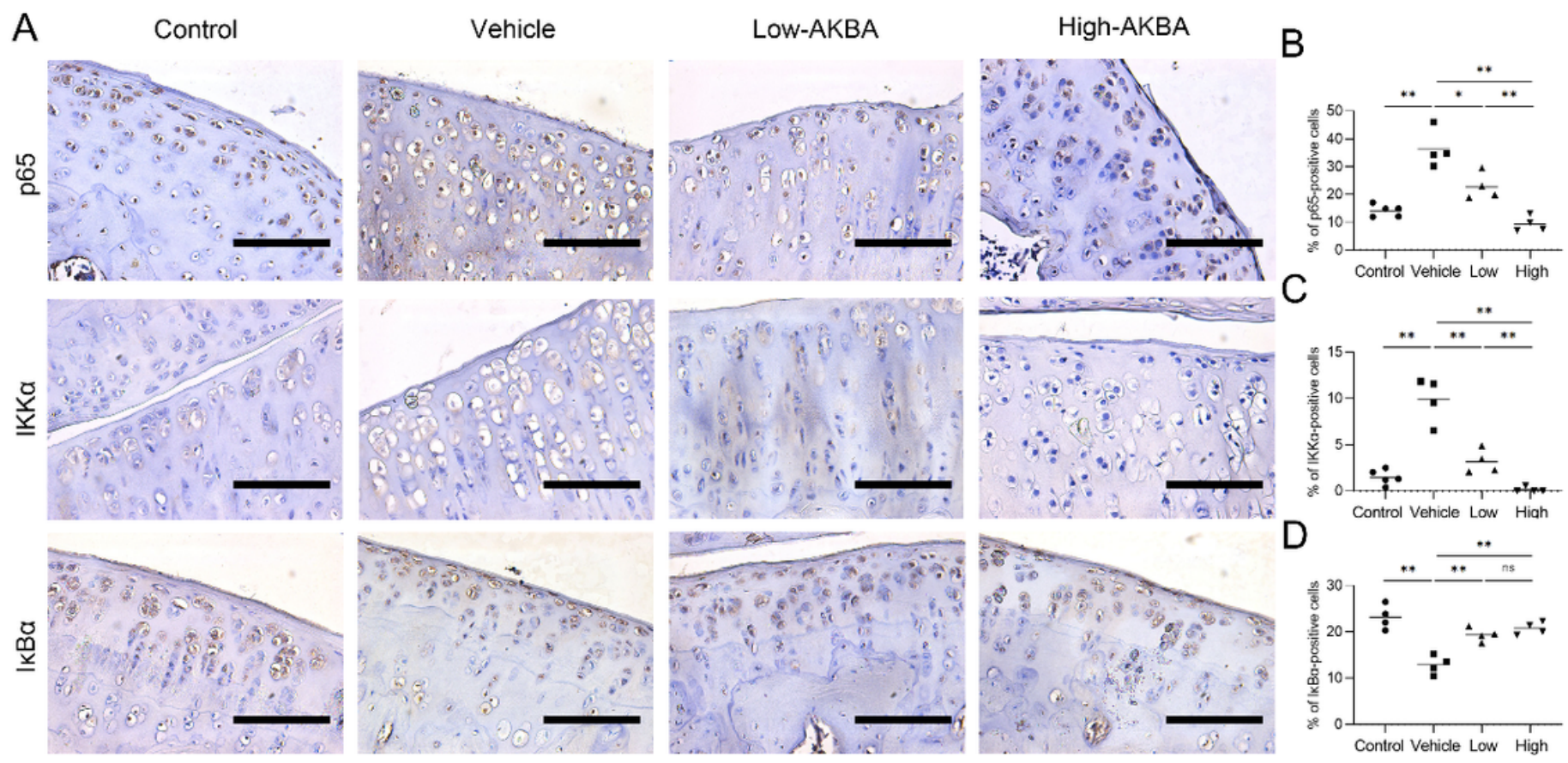

\section{Figure 4}

AKBA intervention inhibited the NF-KB signaling pathway in a rat Hulth-Telhag OA model. (a) IHC analysis of the expression of $\mathrm{p} 65$, IKKa, and IKBa. (b-d) Quantification of the number of positive cells. Both lowAKBA ( $2 \mathrm{mg} / \mathrm{kg}$ every other day) group and high-AKBA ( $8 \mathrm{mg} / \mathrm{kg}$ every other day) group received surgery intervention. $n=6$ per group. Scale bar $=100 \mu \mathrm{m}$. Data are presented as means $\pm S D$. ${ }^{*} P<0.05,{ }^{*} P<0.01$. 
A
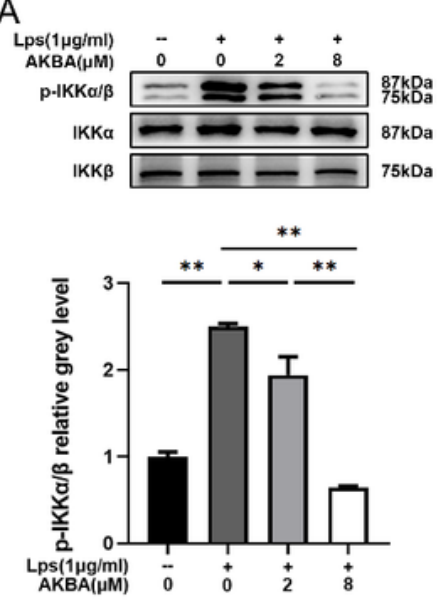

D

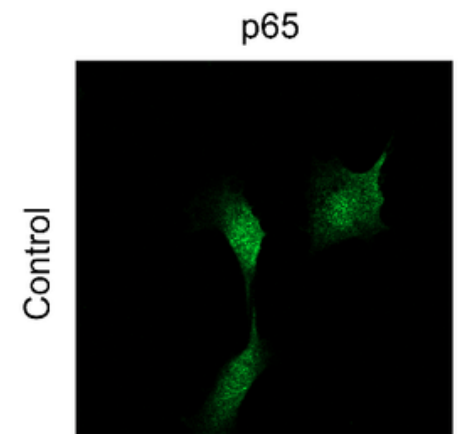

B
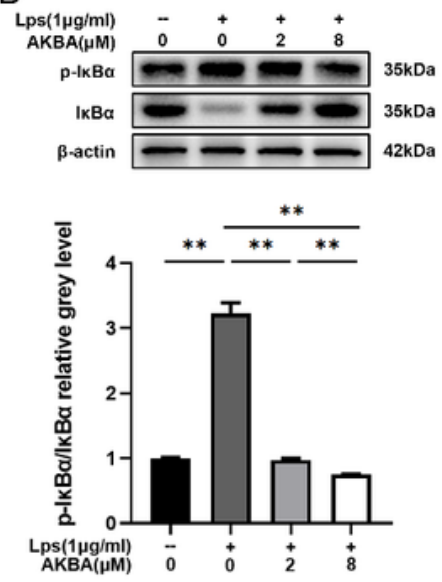

DAPI
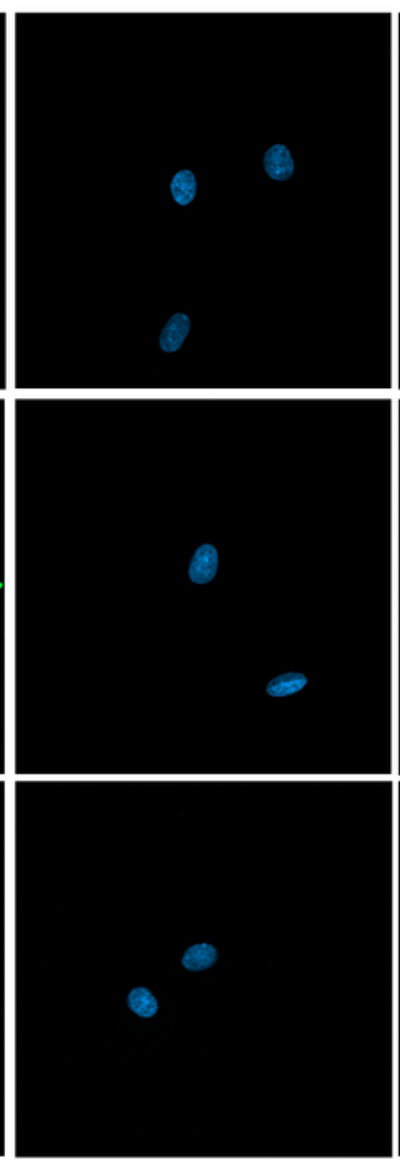

C
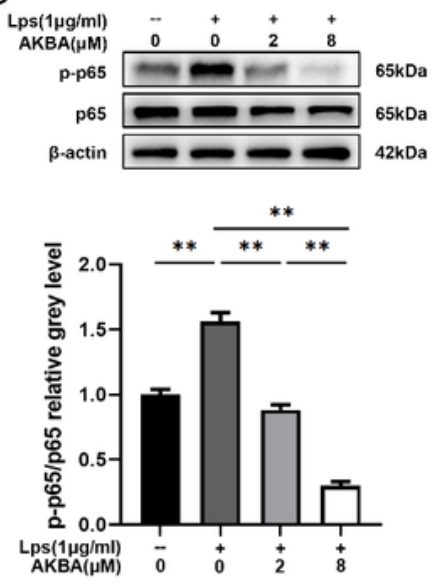

Merge
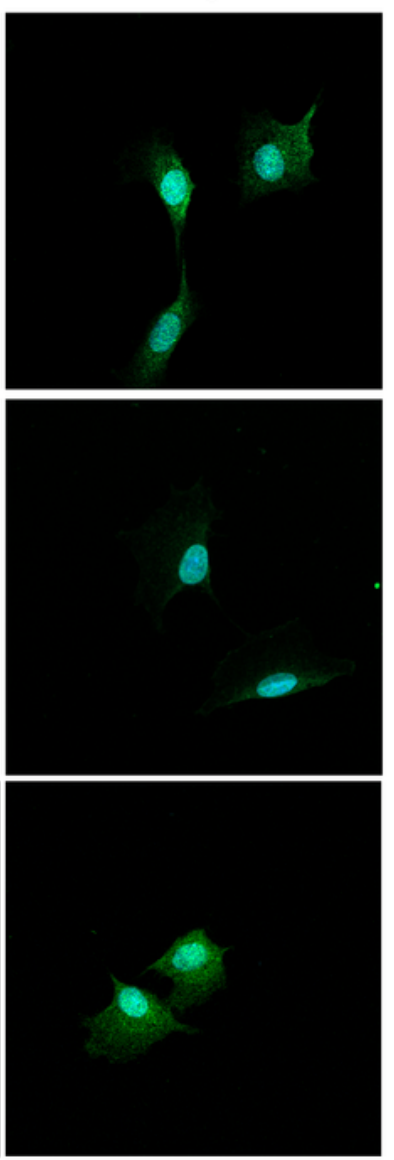

\section{Figure 5}

AKBA intervention attenuated NF-KB activation in LPS-induced rat chondrocytes. (a-c) Western blot analysis of expression levels of $\mathrm{p}-\mathrm{IKK}, \mathrm{IKK}, \mathrm{p}-\mathrm{IKBa}, \mathrm{IKBa}, \mathrm{p}-\mathrm{P} 65$, and P65. The relative grey levels of $\mathrm{p}$ $\mathrm{IKK} / \mathrm{IKK}, \mathrm{p}-\mathrm{IKBa} / \mathrm{IKBa}$, and p-P65/P65. (d) Cellular immunofluorescence of p65 in chondrocytes. Relative grey levels were quantified using Image J. Both low-AKBA $(2 \mu \mathrm{M})$ group and high-AKBA $(8 \mu \mathrm{M})$ group 
contained $1 \mu \mathrm{g} / \mathrm{ml}$ LPS. Dates are presented as means \pm SD of six duplicate experiments. ${ }^{*} P<0.05$, $\star * P<0.01$.

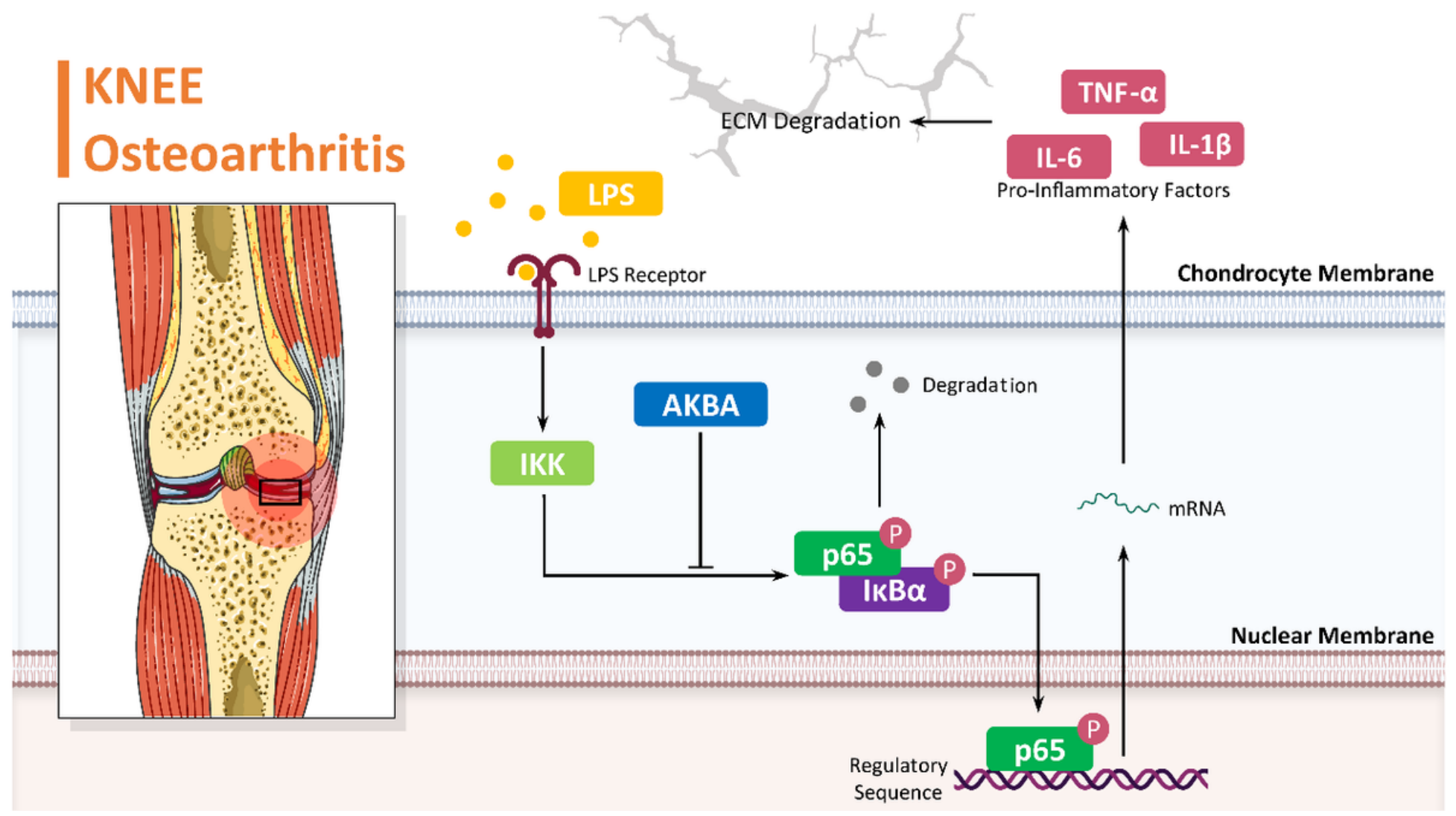

Figure 6

Molecular mechanism of AKBA on LPS-induced rat chondrocytes.

\section{Supplementary Files}

This is a list of supplementary files associated with this preprint. Click to download.

- SupplementaryFiguresS1toS6.docx 